\title{
Classification of continuously transitive circle groups
}

\author{
JAMES GIBLIN \\ VLADIMIR MARKOVIC
}

\begin{abstract}
Let $G$ be a closed transitive subgroup of Homeo $\left(\mathbb{S}^{1}\right)$ which contains a non-constant continuous path $f:[0,1] \rightarrow G$. We show that up to conjugation $G$ is one of the following groups: $\operatorname{SO}(2, \mathbb{R}), \operatorname{PSL}(2, \mathbb{R}), \operatorname{PSL}_{k}(2, \mathbb{R}), \operatorname{Homeo}_{k}\left(\mathbb{S}^{1}\right)$, Homeo($\left(\mathbb{S}^{1}\right)$. This verifies the classification suggested by Ghys in [5]. As a corollary we show that the group $\operatorname{PSL}(2, \mathbb{R})$ is a maximal closed subgroup of $\operatorname{Homeo}\left(\mathbb{S}^{1}\right)$ (we understand this is a conjecture of de la Harpe). We also show that if such a group $G<\operatorname{Homeo}\left(\mathbb{S}^{1}\right)$ acts continuously transitively on $k$-tuples of points, $k>3$, then the closure of $G$ is Homeo( $\left.\mathbb{S}^{1}\right)(\mathrm{cf}[1])$.
\end{abstract}

37E10; 22A05, 54H11

\section{Introduction}

Let Homeo $\left(\mathbb{S}^{1}\right)$ denote the group of orientation preserving homeomorphisms of $\mathbb{S}^{1}$ which we endow with the uniform topology. Let $G$ be a subgroup of Homeo( $\left.\mathbb{S}^{1}\right)$ with the topology induced from Homeo $\left(\mathbb{S}^{1}\right)$. We say that $G$ is transitive if for every two points $x, y \in \mathbb{S}^{1}$, there exists a map $f \in G$, such that $f(x)=y$. We say that a group $G$ is closed if it is closed in the topology of Homeo $\left(\mathbb{S}^{1}\right)$. A continuous path in $G$ is a continuous map $f:[0,1] \rightarrow G$.

Let $\operatorname{SO}(2, \mathbb{R})$ denote the group of rotations of $\mathbb{S}^{1}$ and $\operatorname{PSL}(2, \mathbb{R})$ the group of Möbius transformations. The first main result we prove describes transitive subgroups of $\operatorname{Homeo}\left(\mathbb{S}^{1}\right)$ that contain a non constant continuous path.

Theorem 1.1 Let $G$ be a transitive subgroup of Homeo( $\left.\mathbb{S}^{1}\right)$ which contains a non constant continuous path. Then one of the following mutually exclusive possibilities holds:

(1) $G$ is conjugate to $\operatorname{SO}(2, \mathbb{R})$ in $\operatorname{Homeo}\left(\mathbb{S}^{1}\right)$.

(2) $G$ is conjugate to $\operatorname{PSL}(2, \mathbb{R})$ in $\operatorname{Homeo}\left(\mathbb{S}^{1}\right)$.

(3) For every $f \in \operatorname{Homeo}\left(\mathbb{S}^{1}\right)$ and each finite set of points $x_{1}, \ldots, x_{n} \in \mathbb{S}^{1}$ there exists $g \in G$ such that $g\left(x_{i}\right)=f\left(x_{i}\right)$ for each $i$. 
(4) $G$ is a cyclic cover of a conjugate of $\operatorname{PSL}(2, \mathbb{R})$ in Homeo( $\left.\mathbb{S}^{1}\right)$ and hence conjugate to $\operatorname{PSL}_{k}(2, \mathbb{R})$ for some $k>1$.

(5) $G$ is a cyclic cover of a group satisfying condition 3 above.

Here we write $\operatorname{PSL}_{k}(2, \mathbb{R})$ and $\mathrm{Homeo}_{k}\left(\mathbb{S}^{1}\right)$ to denote the cyclic covers of the groups $\operatorname{PSL}(2, \mathbb{R})$ and Homeo $\left(\mathbb{S}^{1}\right)$ respectively, for some $k \in \mathbb{N}$.

The proof begins by showing that the assumptions of the theorem imply that $G$ is continuously 1-transitive. This means that if we vary points $x, y \in \mathbb{S}^{1}$ in a continuous fashion, then we can choose corresponding elements of $G$ which map $x$ to $y$ that also vary in a continuous fashion. In Theorems 3.8 and 3.10 we show that this leads us to two possibilities, either $G$ is conjugate to $\operatorname{SO}(2, \mathbb{R})$, or $G$ is a cyclic cover of a group which is continuously 2 -transitive.

We then analyse groups which are continuously 2-transitive and show that they are infact all continuously 3-transitive. Furthermore, if such a group is not continuously 4-transitive, we show that it is a convergence group and hence conjugate to $\operatorname{PSL}(2, \mathbb{R})$. On the other hand if it is continuously 4-transitive, then we use an induction argument to show that it is continuously $n$-transitive for all $n \geq 4$. This implies that for every $f \in \operatorname{Homeo}\left(\mathbb{S}^{1}\right)$ and each finite set of points $x_{1}, \ldots, x_{n} \in \mathbb{S}^{1}$ there exists a group element $g$ such that $g\left(x_{i}\right)=f\left(x_{i}\right)$ for each $i$.

The remaining possibilities, namely cases 2 and 3, arise when the aforementioned cyclic cover is trivial.

In the case where the group $G$ is also closed we can use Theorem 1.1 to make the following classification.

Theorem 1.2 Let $G$ be a closed transitive subgroup of Homeo $\left(\mathbb{S}^{1}\right)$ which contains a non constant continuous path. Then one of the following mutually exclusive possibilities holds:

(1) $G$ is conjugate to $\mathrm{SO}(2, \mathbb{R})$ in $\operatorname{Homeo}\left(\mathbb{S}^{1}\right)$.

(2) $G$ is conjugate to $\operatorname{PSL}_{k}(2, \mathbb{R})$ in Homeo( $\left.\mathbb{S}^{1}\right)$ for some $k \geq 1$.

(3) $G$ is conjugate to $\operatorname{Homeo}_{k}\left(\mathbb{S}^{1}\right)$ in $\operatorname{Homeo}\left(\mathbb{S}^{1}\right)$ for some $k \geq 1$.

The above theorem provides the classification of closed, transitive subgroups of Homeo $\left(\mathbb{S}^{1}\right)$ that contain a non-trivial continuous path. This classification was suggested by Ghys for all transitive and closed subgroups of Homeo( $\left.\mathbb{S}^{1}\right)($ See [5]).

One well known problem in the theory of circle groups is to prove that the group of Möbius transformations is a maximal closed subgroup of $\operatorname{Homeo}\left(\mathbb{S}^{1}\right)$. We understand that this is a conjecture of de la Harpe (see [1]). The following theorem follows directly from our work and answers this question. 
Theorem 1.3 PSL $(2, \mathbb{R})$ is a maximal closed subgroup of Homeo $\left(\mathbb{S}^{1}\right)$.

In the following five sections we develop the techniques needed to prove our results. Here we prove the results about the transitivity on $k$-tuples of points. In Section 7 we give the proofs of all the main results stated above.

\section{Continuous Transitivity}

Let $G<\operatorname{Homeo}\left(\mathbb{S}^{1}\right)$ be a transitive group of orientation preserving homeomorphisms of $\mathbb{S}^{1}$. We begin with some definitions which generalize the notion of transitivity.

Set,

$$
P_{n}=\left\{\left(x_{1}, \ldots, x_{n}\right): x_{i} \in \mathbb{S}^{1}, x_{i}=x_{j} \Longleftrightarrow i=j\right\}
$$

to be the set of distinct $n$-tuples of points in $\mathbb{S}^{1}$. Two $n$-tuples

$$
\left(x_{1}, \ldots, x_{n}\right),\left(y_{1}, \ldots, y_{n}\right) \in P_{n}
$$

have matching orientations if there exists $f \in \operatorname{Homeo}\left(\mathbb{S}^{1}\right)$ such that $f\left(x_{i}\right)=y_{i}$ for each $i$.

Definition 2.1 $G$ is $n$-transitive if for every pair $\left(x_{1}, \ldots, x_{n}\right),\left(y_{1}, \ldots, y_{n}\right) \in P_{n}$ with matching orientations there exists $g \in G$ such that $g\left(x_{i}\right)=y_{i}$ for each $i$.

Definition 2.2 $G$ is uniquely $n$-transitive if it is $n$-transitive and for each pair $\left(x_{1}, \ldots, x_{n}\right),\left(y_{1}, \ldots, y_{n}\right) \in P_{n}$ with matching orientations there is exactly one element $g \in G$ such that $g\left(x_{i}\right)=y_{i}$. Equivalently, the only element of $G$ fixing $n$ distinct points is the identity.

Endow $\mathbb{S}^{1}$ with the standard topology and $P_{n}$ with the topology it inherits as a subspace of the $n$-fold Cartesian product $\mathbb{S}^{1} \times \cdots \times \mathbb{S}^{1}$. These are metric topologies. With the topology on $P_{n}$ being induced by the distance function

$$
d_{P_{n}}\left(\left(x_{1}, \ldots, x_{n}\right),\left(y_{1}, \ldots, y_{n}\right)\right)=\max \left\{d_{\mathbb{S}_{1}}\left(x_{i}, y_{i}\right): i=1, \ldots, n\right\},
$$

where $d_{\mathbb{S}^{1}}$ is the standard Euclidean distance function on $\mathbb{S}^{1}$.

Endow $G$ with the uniform topology. This is also a metric topology, induced by the distance function,

$$
d_{G}\left(g_{1}, g_{2}\right)=\sup \left\{\max \left\{d_{\mathbb{S}^{1}}\left(g_{1}(x), g_{2}(x)\right), d_{\mathbb{S}^{1}}\left(g_{1}^{-1}(x), g_{2}^{-1}(x)\right)\right\}: x \in \mathbb{S}^{1}\right\}
$$

A path in a topological space $X$ is a continuous map $\gamma:[0,1] \rightarrow X$. If $\mathcal{X}:[0,1] \rightarrow P_{n}$ is a path in $P_{n}$ we will write $x_{i}(t)=\pi_{i} \circ \mathcal{X}(t)$, where $\pi_{i}$ is projection onto the $i$-th 
component of $\mathbb{S}^{1} \times \cdots \times \mathbb{S}^{1}$, so that we can write $\mathcal{X}(t)=\left(x_{1}(t), \cdots, x_{n}(t)\right)$. We will call a pair of paths $\mathcal{X}, \mathcal{Y}:[0,1] \rightarrow P_{n}$ compatible if there exists a path $h:[0,1] \rightarrow$ $\operatorname{Homeo}\left(\mathbb{S}^{1}\right)$ with $h(t)\left(x_{i}(t)\right)=y_{i}(t)$ for each $i$ and $t$.

Definition 2.3 $G$ is continuously $n$-transitive if for every compatible pair of paths $\mathcal{X}, \mathcal{Y}:[0,1] \rightarrow P_{n}$ there exists a path $g:[0,1] \rightarrow G$ with the property that $g(t)\left(x_{i}(t)\right)=$ $y_{i}(t)$ for each $i$ and $t$.

Definition 2.4 A continuous deformation of the identity in $G$ is a non constant path of homeomorphisms $f_{t} \in G$ for $t \in[0,1]$ with $f_{0}=\mathrm{id}$.

We have the following lemma.

Lemma 2.5 For $n \geq 2$ the following are equivalent:

(1) $G$ is continuously $n$-transitive.

(2) $G$ is continuously $n-1-$ transitive and the following holds. For every $n-1-t u p l e$ $\left(a_{1}, \ldots, a_{n-1}\right) \in P_{n-1}$ and $x \in \mathbb{S}^{1} \backslash\left\{a_{1}, \ldots, a_{n-1}\right\}$ there exists a continuous map $F_{x}: I_{x} \rightarrow G$ satisfying the following conditions,

(a) $F_{x}(y)$ fixes $a_{1}, \ldots, a_{n-1}$ for all $y \in I_{x}$

(b) $\left(F_{x}(y)\right)(x)=y$ for all $y \in I_{x}$

(c) $F_{x}(x)=\mathrm{id}$

where $I_{x}$ is the component of $\mathbb{S}^{1} \backslash\left\{a_{1}, \ldots, a_{n-1}\right\}$ containing $x$.

(3) $G$ is continuously $n-1$-transitive and there exists $\left(a_{1}, \ldots, a_{n-1}\right) \in P_{n-1}$ with the following property. There is a component $I$ of $\mathbb{S}^{1} \backslash\left\{a_{1}, \ldots, a_{n-1}\right\}$, a point $\tilde{x} \in I$ and a continuous map $F_{\tilde{x}}: I \rightarrow G$ satisfying the following conditions,

(a) $F_{\tilde{x}}(y)$ fixes $a_{1}, \ldots, a_{n-1}$ for all $y \in I$

(b) $\left(F_{\tilde{x}}(y)\right)(\tilde{x})=y$ for all $y \in I$

(c) $F_{\tilde{x}}(\tilde{x})=\mathrm{id}$.

(4) $G$ is continuously $n-1$-transitive and there exists $\left(a_{1}, \ldots, a_{n-1}\right) \in P_{n-1}$ with the following property. There is a component $I$ of $\mathbb{S}^{1} \backslash\left\{a_{1}, \ldots, a_{n-1}\right\}$, such that for each $x \in I$ there exists a continuous deformation of the identity $f_{t}$, satisfying $f_{t}\left(a_{i}\right)=a_{i}$ for each $t$ and $i$ and $f_{t}(x) \neq x$ for some $t$.

Proof We start by showing $[1 \Rightarrow 4]$. As $G$ is continuously $n$-transitive, it will automatically be continuously $n-1$ transitive. Take $\left(a_{1}, \ldots, a_{n-1}\right) \in P_{n-1}$ and $x \in \mathbb{S}^{1} \backslash\left\{a_{1}, \ldots, a_{n-1}\right\}$. Let $I_{x}$ be the component of $\mathbb{S}^{1} \backslash\left\{a_{1}, \ldots, a_{n-1}\right\}$ which contains $x$. Take $y \in I_{x} \backslash\{x\}$ and let $x_{t}$ be an injective path in $I_{x}$ with $x_{0}=x$ and $x_{1}=y$. 
Let $\mathcal{X}:[0,1] \rightarrow P_{n}$ be the constant path defined by $\mathcal{X}(t)=\left(a_{1}, \ldots, a_{n-1}, x_{0}\right)$ and let $\mathcal{Y}:[0,1] \rightarrow P_{n}$ be the path defined by $\mathcal{Y}(t)=\left(a_{1}, \ldots, a_{n-1}, x_{t}\right)$. Then since $x_{t} \in I_{x}$ for every time $t$ these form an compatible pair of paths. Consequently, there exists a path $g_{t} \in G$ which fixes each $a_{i}$ and such that $g_{t}(x)=\left(x_{t}\right)$. Defining $f_{t}=g_{t} \circ\left(g_{0}^{-1}\right)$ gives us the required continuous deformation of the identity.

We now show that $[4 \Rightarrow 3]$. For $\tilde{x} \in I$ set $K_{\tilde{x}}$ to be the set of points $x \in I$ for which there is a path of homeomorphisms $f_{t} \in G$ satisfying,

(1) $f_{0}=\mathrm{id}$

(2) $f_{t}\left(a_{i}\right)=a_{i}$ for each $i$ and $t$

(3) $f_{1}(\tilde{x})=x$.

Obviously, $K_{\tilde{x}}$ will be a connected subset of $I$ and hence an interval for each $\tilde{x} \in I$. Choose $\tilde{x} \in I$ and take $x \in K_{\tilde{x}}$. Let $f_{t}$ and $g_{t}$ be continuous deformations of the identity which fix the $a_{i}$ for all $t$ and such that $f_{t_{0}}(x) \neq x$ for some $t_{0} \in(0,1]$ and $g_{1}(\tilde{x})=x . f_{t}$ exists by the assumptions of condition 4 . and $g_{t}$ exists because $x \in K_{\tilde{x}}$. The following paths show that the interval between $f_{t_{0}}(x)$ and $\left(f_{t_{0}}\right)^{-1}(x)$ is contained in $K_{\tilde{x}}$ :

$$
\begin{gathered}
h_{1}(t)= \begin{cases}g_{2 t} & t \in[0,1 / 2] \\
f_{t_{0}(2 t-1)} \circ g_{1} & t \in[1 / 2,1]\end{cases} \\
h_{2}(t)= \begin{cases}g_{2 t} & t \in[0,1 / 2] \\
\left(f_{t_{0}(2 t-1)}\right)^{-1} \circ g_{1} & t \in[1 / 2,1]\end{cases}
\end{gathered}
$$

As $x$ is contained in this interval and cannot be equal to either of its endpoints we see that $K_{\tilde{x}}$ is open for every $\tilde{x} \in I$. On the other hand, $\tilde{x} \in K_{\tilde{x}}$ for each $\tilde{x} \in I$ and if $x_{1} \in K_{x_{2}}$ then $K_{x_{1}}=K_{x_{2}}$. Consequently, the sets $\left\{K_{\tilde{x}}: \tilde{x} \in I\right\}$ form a partition of $I$ and hence $K_{\tilde{x}}=I$ for every $\tilde{x} \in I$.

We now construct the map $F_{\tilde{x}}$. To do this, take a nested sequence of intervals $\left[x_{n}, y_{n}\right]$ containing $\tilde{x}$ for each $n$ and such that $x_{n}, y_{n}$ converge to the endpoints of $I$ as $n \rightarrow \infty$. We define $F_{\tilde{x}}$ inductively on these intervals. Since $K_{\tilde{x}}=I$ we can find a path of homeomorphisms $f_{t} \in G$ satisfying,

(1) $f_{0}=\mathrm{id}$

(2) $f_{t}\left(a_{i}\right)=a_{i}$ for each $i$ and $t$

(3) $f_{1}(\tilde{x})=x_{1}$.

We now show that there exists a path $\overline{f_{t}} \in G$, which also satisfies the above, but with the additional condition that the path $\overline{f_{t}}(\tilde{x})$ is simple.

To see this, let $\left[x^{*}, \tilde{x}\right]$ be the largest subinterval of $\left[x_{1}, \tilde{x}\right]$ for which there exists a path $\overline{f_{t}} \in G$ which satisfies, 
(1) $\overline{f_{0}}=\mathrm{id}$

(2) $\bar{f}_{t}\left(a_{i}\right)=a_{i}$ for each $i$ and $t$

(3) $\overline{f_{1}}(\tilde{x})=x^{*}$

(4) $\overline{f_{t}}(\tilde{x})$ is simple.

We want to show that $x^{*}=x_{1}$. Assume for contradiction that $x^{*} \neq x_{1}$. Then since $x^{*} \in\left[x_{1}, \tilde{x}\right]$ there exists $s \in[0,1]$ such that $f_{s}(\tilde{x})=x^{*}$ and for small $\epsilon>0$, we have that $f_{s+\epsilon}(\tilde{x}) \notin\left[x^{*}, \tilde{x}\right]$. Then if we concatenate the path $\overline{f_{t}}$ with $f_{s+\epsilon} \circ f_{s}^{-1} \circ \overline{f_{1}}$ for small $\epsilon$ we can construct a simple path satisfying the same conditions as $\bar{f}_{t}$ but on a interval strictly bigger than $\left[x^{*}, \tilde{x}\right]$, this contradicts the maximality of $x^{*}$ and we deduce that $x^{*}=x_{1}$.

We can use the path $\bar{f}_{t}$ to define a map $F_{\tilde{x}}:\left[x_{1}, y_{1}\right] \rightarrow G$ satisfying,

(1) $F_{\tilde{x}}^{1}(y)$ fixes each $a_{i}$ for each $y \in I$

(2) $\left(F_{\tilde{x}}^{1}(y)\right)(\tilde{x})=y$ for all $y \in I$

(3) $F_{\tilde{x}}(\tilde{x})=\mathrm{id}$.

by taking paths of homeomorphisms that move $\tilde{x}$ to $x_{1}$ and $y_{1}$ along simple paths in $S^{1}$.

Now assume we have defined a map $F_{\tilde{x}}^{k}:\left[x_{k}, y_{k}\right] \rightarrow G$ satisfying,

(1) $F_{\tilde{x}}^{k}(y)$ fixes each $a_{i}$ for each $y \in I$

(2) $\left(F_{\tilde{x}}^{k}(y)\right)(\tilde{x})=y$ for all $y \in I$

(3) $F_{\tilde{x}}^{k}(\tilde{x})=\mathrm{id}$.

We can use the same argument used to produce $F_{\tilde{x}}$ to show that there exists a map $\mathfrak{F}_{x_{k}}:\left[x_{k+1}, x_{k}\right] \rightarrow G$ such that $\mathfrak{F}_{x_{k}}(x)$ fixes the $a_{i}$ for each $x, \mathfrak{F}_{x_{k}}\left(x_{k}\right)=$ id and $\left(\mathfrak{F}_{x_{k}}(x)\right)\left(x_{k}\right)=x$. Similarly there exists a map $\mathfrak{F}_{y_{k}}:\left[y_{k}, y_{k+1}\right] \rightarrow G$ such that $\mathfrak{F}_{y_{k}}(x)$ fixes the $a_{i}$ for each $x, \mathfrak{F}_{y_{k}}\left(y_{k}\right)=\mathrm{id}$ and $\left(\mathfrak{F}_{y_{k}}(x)\right)\left(y_{k}\right)=x$.

This allows us to define, $F_{\tilde{x}}^{k+1}:\left[x_{k+1}, y_{k+1}\right] \rightarrow G$ by:

$$
F_{\tilde{x}}^{k+1}(x)= \begin{cases}F_{\tilde{x}}^{k}(x) & x \in\left[x_{k}, y_{k}\right] \\ \left(\mathfrak{F}_{x_{k}}(x)\right) \circ F_{\tilde{x}}^{k}\left(x_{k}\right) & x \in\left[x_{k+1}, x_{k}\right] \\ \left(\mathfrak{F}_{y_{k}}(x)\right) \circ F_{\tilde{x}}^{k}\left(y_{k}\right) & x \in\left[y_{k}, y_{k+1}\right]\end{cases}
$$

Inductively, we can now define the full map $F_{\tilde{x}}: I \rightarrow G$.

We now show that $[3 \Rightarrow 2]$. So take $x^{\prime} \in I$ with $x^{\prime} \neq \tilde{x}$ and define $F_{x^{\prime}}: I \rightarrow G$ by

$$
F_{x^{\prime}}(y)=F_{\widetilde{x}}(y) \circ\left(F_{\widetilde{x}}\left(x^{\prime}\right)\right)^{-1}
$$

Then $F_{x^{\prime}}$ satisfies, 
(1) $F_{x^{\prime}}(y)$ fixes $a_{1}, \ldots, a_{n-1}$ for all $y \in I$

(2) $\left(F_{x^{\prime}}(y)\right)\left(x^{\prime}\right)=y$ for all $y \in I$

(3) $F_{x^{\prime}}\left(x^{\prime}\right)=\mathrm{id}$.

Moreover, we can use (1) to define a map $F: I \times I \rightarrow G$ which is continuous in each variable and satisfies,

(1) $F(x, y)$ fixes $a_{1}, \ldots, a_{n-1}$ for all $x, y \in I$

(2) $(F(x, y))(x)=y$ for all $x, y \in I$

(3) $F(x, x)=$ id for all $x \in I$.

Now take $x^{\prime}$ to be a point in $\mathbb{S}^{1} \backslash I \cup\left\{a_{1}, \ldots, a_{n-1}\right\}$ and let $I^{\prime}$ be the component of $\mathbb{S}^{1} \backslash\left\{a_{1}, \ldots, a_{n-1}\right\}$ which contains $x^{\prime}$. Then since $G$ is continuously $n-1$-transitive there exists $g \in G$ which permutes the $a_{i}$ so that $g(I)=I^{\prime}$. Define $F_{x^{\prime}}: I^{\prime} \rightarrow G$ by

$$
F_{x^{\prime}}(y)=g \circ F_{g^{-1}\left(x^{\prime}\right)}\left(g^{-1}(y)\right) \circ g^{-1}
$$

for $y \in I^{\prime}$. Then $F_{x^{\prime}}$ satisfies,

(1) $F_{x^{\prime}}(y)$ fixes $a_{1}, \ldots, a_{n-1}$ for all $y \in I$

(2) $\left(F_{x^{\prime}}(y)\right)\left(x^{\prime}\right)=y$ for all $y \in I^{\prime}$

(3) $F_{x^{\prime}}\left(x^{\prime}\right)=$ id.

Now let $\left(b_{1}, \ldots, b_{n-1}\right) \in P_{n-1}$ have the same orientation as $\left(a_{1}, \ldots, a_{n-1}\right)$ then since $G$ is continuously $n-1$-transitive there exists $g \in G$ so that $g\left(a_{i}\right)=b_{i}$ for each $i$. Let $x^{\prime} \in \mathbb{S}^{1} \backslash\left\{b_{1}, \ldots, b_{n-1}\right\}$ and let $I^{\prime}$ be the component of $\mathbb{S}^{1} \backslash\left\{b_{1}, \ldots, b_{n-1}\right\}$ in which it lies. Define $F_{x^{\prime}}: I^{\prime} \rightarrow G$ by

$$
F_{x^{\prime}}(y)=g \circ F_{g^{-1}\left(x^{\prime}\right)}\left(g^{-1}(y)\right) \circ g^{-1}
$$

for $y \in I^{\prime}$. Then $F_{x^{\prime}}$ satisfies,

(1) $F_{x^{\prime}}(y)$ fixes $b_{1}, \ldots, b_{n-1}$ for all $y \in I$

(2) $\left(F_{x^{\prime}}(y)\right)\left(x^{\prime}\right)=y$ for all $y \in I^{\prime}$

(3) $F_{x^{\prime}}\left(x^{\prime}\right)=\mathrm{id}$

and we have that $[3 \Rightarrow 2]$

Finally we have to show that $[2 \Rightarrow 1]$. Let $\mathcal{X}, \mathcal{Y}:[0,1] \rightarrow P_{n}$ be an compatible pair of paths. We define $\mathcal{X}^{\prime}:[0,1] \rightarrow P_{n-1}$ by

$$
\mathcal{X}^{\prime}(t)=\left(x_{1}(t), \ldots, x_{n-1}(t)\right)
$$


and $\mathcal{Y}^{\prime}:[0,1] \rightarrow P_{n-1}$ by

$$
\mathcal{Y}^{\prime}(t)=\left(y_{1}(t), \ldots, y_{n-1}(t)\right)
$$

Notice that $\mathcal{X}^{\prime}$ and $\mathcal{Y}^{\prime}$ will also be a compatible pair of paths. Furthermore, as $G$ is continuously $n-1$-transitive there will exist a path $g^{\prime}:[0,1] \rightarrow G$ such that $g^{\prime}(t)\left(x_{i}(t)\right)=y_{i}(t)$ for $1 \leq i \leq n-1$.

The paths $\mathcal{X}^{\prime}, \mathcal{Y}^{\prime}:[0,1] \rightarrow P_{n-1}$ will also be compatible with the constant paths,

$$
\begin{gathered}
\mathcal{X}_{0}^{\prime}:[0,1] \rightarrow P_{n-1} \\
\mathcal{X}_{0}^{\prime}(t)=\mathcal{X}^{\prime}(0)
\end{gathered}
$$

and

$$
\begin{gathered}
\mathcal{Y}_{0}^{\prime}:[0,1] \rightarrow P_{n-1} \\
\mathcal{Y}_{0}^{\prime}(t)=\mathcal{Y}^{\prime}(0)
\end{gathered}
$$

respectively. So that there exist paths $g_{x}^{\prime}, g_{y}^{\prime}:[0,1] \rightarrow G$ with $g_{x}^{\prime}\left(x_{i}(0)\right)=x_{i}(t)$ and $g_{y}^{\prime}\left(y_{i}(0)\right)=y_{i}(t)$ for $1 \leq i \leq n-1$. Furthermore, by pre composing with $\left(g_{x}^{\prime}(0)\right)^{-1}$ and $\left(g_{y}^{\prime}(0)\right)^{-1}$ if necessary, we can assume that $g_{x}^{\prime}(0)=g_{y}^{\prime}(0)=\mathrm{id}$.

We now construct a path $g_{x}:[0,1] \rightarrow G$ which satisfies,

$$
g_{x}(t)\left(x_{i}(0)\right)=x_{i}(t)
$$

for $1 \leq i \leq n$. To do this let $I$ be the component of $\mathbb{S}^{1} \backslash\left\{x_{1}(0), \ldots, x_{n-1}(0)\right\}$ containing $x_{n}(0)$. By assumption we have a continuous map $F_{x_{n}(0)}: I \rightarrow G$ satisfying

(1) $F_{x_{n}(0)}(y)$ fixes $x_{1}(0), \ldots, x_{n-1}(0)$ for all $y \in I$

(2) $\left(F_{x_{n}(0)}(y)\right)(x)=y$ for all $y \in I$

(3) $F_{x_{n}(0)}(x)=$ id.

Define $g_{x}:[0,1] \rightarrow G$ by

$$
g_{x}(t)=g_{x}^{\prime}(t) \circ\left(F_{x_{n}(0)}\left(\left(g_{x}^{\prime}(t)\right)^{-1}\left(x_{n}(t)\right)\right)\right)^{-1} .
$$

Then $g_{x}(t)\left(x_{i}(0)\right)=x_{i}(t)$ for $1 \leq i \leq n$. We can repeat this process with $g_{y}^{\prime}$ to construct a path $g_{y}:[0,1] \rightarrow G$ satisfying $g_{y}(t)\left(y_{i}(0)\right)=y_{i}(t)$ for $1 \leq i \leq n$.

The map $g^{\prime}(0)$ which we defined earlier will map $x_{i}(0)$ to $y_{i}(0)$ for $1 \leq i \leq n-1$. Moreover, $g^{\prime}(0)\left(x_{n}(0)\right)$ will lie in the same component of $\mathbb{S}^{1} \backslash\left\{y_{1}(0), \ldots y_{n-1}(0)\right\}$ as $y_{n}(0)$. So we have a map $F_{g^{\prime}(0)\left(x_{n}(0)\right)}\left(y_{n}(0)\right)$ which maps $g^{\prime}(0)\left(x_{n}(0)\right)$ to $y_{n}(0)$ and fixes the other $y_{i}(0)$. Putting all of this together allows us to define $g:[0,1] \rightarrow G$ by

$$
g(t)=g_{y}(t) \circ F_{g^{\prime}(0)\left(x_{n}(0)\right)}\left(y_{n}(0)\right) \circ g^{\prime}(0) \circ\left(g_{x}(t)\right)^{-1} .
$$


This is a path in $G$ which satisfies $g_{t}\left(x_{i}(t)\right)=y_{i}(t)$ for each $i$ and $t$. Since we can do this for any two compatible paths, $G$ is continuously $n$-transitive and we have shown that $[2 \Rightarrow 1]$.

Proposition 2.6 If $G$ is 1-transitive and there exists a continuous deformation of the identity $f_{t}:[0,1] \rightarrow G$ in $G$, then $G$ is continuously $1-$ transitive.

Proof Let $x_{0} \in \mathbb{S}^{1}$ be such that $f_{t_{0}}\left(x_{0}\right) \neq x_{0}$ for some $t_{0} \in[0,1]$. Take $x \in \mathbb{S}^{1}$ then there exists $g \in G$ such that $g(x)=x_{0}$. Consequently, $g^{-1} \circ f_{t} \circ g$ is a continuous deformation of the identity which doesn't fix $x$ for some $t$. Since these deformations exist for each $x \in \mathbb{S}^{1}$ the proof follows in exactly the same way as $[4 \Rightarrow 1]$ from the proof of Lemma 2.5.

From now on we will assume that $G$ contains a continuous deformation of the identity, and hence is continuously 1 -transitive.

\section{The set $J_{x}$}

Definition 3.1 For $x \in \mathbb{S}^{1}$ we define $J_{x}$ to be the set of points $y \in \mathbb{S}^{1}$ which satisfy the following condition. There exists a continuous deformation of the identity $f_{t} \in G$ which fixes $x$ for all $t$ and such that $f_{t_{0}}(y) \neq y$ for some $t_{0} \in[0,1]$.

It follows directly from this definition that $x \notin J_{x}$.

Lemma 3.2 $J_{f(x)}=f\left(J_{x}\right)$ for every $f \in G$ and $x \in \mathbb{S}^{1}$.

Proof Let $y \in J_{f(x)}$ and let $f_{t}$ be the corresponding continuous deformation of the identity with $f_{t_{0}}(y) \neq y$. Then $f^{-1} \circ f_{t} \circ f$ is also a continuous deformation of the identity which now fixes $x$, and for which $f_{t_{0}}\left(f^{-1}(y)\right) \neq f^{-1}(y)$. This means that $f^{-1}(y) \in J_{x}$ and hence $y \in f\left(J_{x}\right)$ so that $J_{f(x)} \subset f\left(J_{x}\right)$. The other inclusion is an identical argument.

Lemma 3.3 $J_{x}$ is open for every $x \in \mathbb{S}^{1}$.

Proof Let $y \in J_{x}$ and take $f_{t}$ to be the corresponding continuous deformation of the identity with $f_{t_{0}}(y) \neq y$ for some $t_{0} \in[0,1]$. Then since $f_{t_{0}}$ is continuous there exists a neighborhood $U$ of $y$ such that $f_{t_{0}}(z) \neq z$ for all $z \in U$. This implies that $U \subset J_{x}$ and hence that $J_{x}$ is open. 
Lemma 3.4 $J_{x}=\varnothing$ for every $x \in \mathbb{S}^{1}$ or $J_{x}$ has a finite complement for every $x \in \mathbb{S}^{1}$.

To prove this lemma we will use the Hausdorff maximality Theorem which we now recall.

Definition 3.5 A set $\mathcal{P}$ is partially ordered by a binary relation $\leq$ if,

(1) $a \leq b$ and $b \leq c$ implies $a \leq c$

(2) $a \leq a$ for every $a \in \mathcal{P}$

(3) $a \leq b$ and $b \leq a$ implies that $a=b$.

Definition 3.6 A subset $\mathcal{Q}$ of a partially ordered set $\mathcal{P}$ is totally ordered if for every pair $a, b \in \mathcal{Q}$ either $a \leq b$ or $b \leq a$. A totally ordered subset $\mathcal{Q} \subset \mathcal{P}$ is maximal if for any member $a \in \mathcal{P} \backslash \mathcal{Q}, \mathcal{Q} \cup\{a\}$ is not totally ordered.

Theorem 3.7 (Hausdorff Maximality Theorem) Every nonempty partially ordered set contains a maximal totally ordered subset.

We now prove Lemma 3.4.

Proof Assume that there exists $x \in \mathbb{S}^{1}$ for which $J_{x}=\varnothing$. Then for every $y \in \mathbb{S}^{1}$ there exists a map $g \in G$ such that $g(x)=y$. Consequently,

$$
J_{y}=J_{g(x)}=g\left(J_{x}\right)=g(\varnothing)=\varnothing
$$

for every $y \in \mathbb{S}^{1}$.

Assume that $J_{x} \neq \varnothing$ for every $x \in \mathbb{S}^{1}$ and let $S_{x}=\mathbb{S}^{1} \backslash J_{x}$ denote the complement of $J_{x}$. This means that $S_{x}$ consists of the points $y \in \mathbb{S}^{1}$ such every continuous deformation of the identity which fixes $x$ also fixes $y$. The set $\mathcal{P}=\left\{S_{x}: x \in \mathbb{S}^{1}\right\}$ is partially ordered by inclusion so that by Theorem 3.7 there exists a maximal totally ordered subset, $\mathcal{Q}=\left\{S_{x}: x \in A\right\}$, where $A$ is the appropriate subset of $\mathbb{S}^{1}$.

If we set $\mathcal{S}=\bigcap_{x \in A} S_{x}$ then we have the following:

(1) $\mathcal{S} \neq \varnothing$

(2) if $x \in \mathcal{S}$ then $S_{x}=\mathcal{S}$. 
(1) follows from the fact that $\mathcal{S}$ is the intersection of a descending family of compact sets, and hence is nonempty.

To see that (2) is also true, fix $x \in \mathcal{S}$. Then from the definition of $\mathcal{S}$, we will have $x \in S_{a}$ for each $a \in A$. In other words, if we take $a \in A$, then every continuous deformation of the identity which fixes $a$ will also fix $x$. Furthermore, if $y \in S_{x}$ then every continuous deformation of the identity which fixes $a$ not only fixes $x$ but $y$ too, so that $S_{x} \subset S_{a}$. This is true for every $a \in A$ so that $S_{x} \subset \mathcal{S}$. On the other hand, by the maximality of $\mathcal{Q}$, it must contain $S_{x}$. Consequently, if $x \in \mathcal{S}$ then $S_{x}=\mathcal{S}$.

Fix $x_{0} \in \mathcal{S}$ and assume for contradiction that $S_{x_{0}}$ is infinite. Take a sequence $x_{n} \in S_{x_{0}}$ and let $x_{n_{k}}$ be a convergent subsequence with limit $x^{\prime}$. This limit will also be in $S_{x_{0}}$ as it is closed. As $J_{x_{0}}$ is a nonempty open subset of $\mathbb{S}^{1}$ it will contain an interval $(a, b)$ with $a, b \in S_{x_{0}}$. Take maps $g_{a}, g_{b} \in G$ so that $g_{a}\left(x^{\prime}\right)=a$ and $g_{b}\left(x^{\prime}\right)=b$. Since $x^{\prime}, a \in S_{x_{0}}$ we have that,

$$
g_{a}\left(S_{x_{0}}\right)=g_{a}\left(S_{x^{\prime}}\right)=S_{g_{a}\left(x^{\prime}\right)}=S_{a}=S_{x_{0}}
$$

and similarly for $g_{b}$. As a result $g_{a}\left(x_{n}\right), g_{b}\left(x_{n}\right) \in S_{x_{0}}$ for each $n$, but $g_{a}, g_{b}$ are orientation preserving homeomorphisms so that at least one of these points will lie in $(a, b)$, a contradiction.

We have shown that $S_{x_{0}}$ is finite. If we now take any other point $x \in \mathbb{S}^{1}$ then there exists a map $g \in G$ such that $g\left(x_{0}\right)=x$. This means that the set $S_{x}=S_{g\left(x_{0}\right)}=g\left(S_{x_{0}}\right)$ will also be finite and we are done.

Theorem 3.8 If $J_{x}=\varnothing$ for all $x \in \mathbb{S}^{1}$ then $G$ is conjugate in Homeo( $\left(\mathbb{S}^{1}\right)$ to the group of rotations $\mathrm{SO}(2, \mathbb{R})$.

We require the following lemma for the proof of this Theorem.

Lemma 3.9 If $f: \mathbb{R} \rightarrow \mathbb{R}$ is a homeomorphism which conjugates translations to translations, then it is an affine map.

Proof Let $f$ be a homeomorphism which conjugates translations to translations and set $f_{1}=T \circ f$ where $T$ is the translation that sends $f(0)$ to 0 . Then $f_{1}$ fixes 0 and also conjugates translations to translations. In particular there exists $\alpha$ such that $f_{1}$ conjugates $x \mapsto x+1$ to the map $x \mapsto x+\alpha$. Notice that $\alpha \neq 0$ since the identity is only conjugate to itself.

Now define $f_{2}=f_{1} \circ M_{\alpha}$ where $M_{\alpha}(x)=\alpha x$. A simple calculation shows that $f_{2}$ conjugates $x \mapsto x+1$ to itself and conjugates translations to translations. Since $f_{2}$ 
fixes 0 and conjugates $x \mapsto x+1$ to itself, we deduce that it must fix all the integer points.

Now, for $n \in \mathbb{N}$ let $\gamma \in \mathbb{R}$ be such that $\left(f_{2}\right)^{-1} \circ T_{1 / n} \circ f_{2}=T_{\gamma}$ where $T_{\alpha}(x)=x+\alpha$. It follows that,

$$
T_{1}=\left(f_{2}\right)^{-1} \circ\left(T_{1 / n}\right)^{n} \circ f_{2}=\left(\left(f_{2}\right)^{-1} \circ T_{1 / n} \circ f_{2}\right)^{n}=\left(T_{\gamma}\right)^{n}
$$

so that $\gamma=1 / n$ and $\left(f_{2}\right)^{-1} \circ T_{1 / n} \circ f_{2}=T_{1 / n}$ for every $n \in \mathbb{N}$. Combining this with the fact that $f_{2}$ fixes 0 , we see that $f_{2}$ must fix all the rational points and hence is the identity. This implies that $f_{1}$ and hence $f$ are affine.

We can now prove Theorem 3.8.

Proof Let $\widehat{G}<G$ denote the path component of the identity in $G$. We are going to show that $\widehat{G}$ is a compact group. Proposition 4.1 in [5] will then imply that it is conjugate in Homeo( $\left(\mathbb{S}^{1}\right)$ to a subgroup of $\operatorname{SO}(2, \mathbb{R})$. Moreover, as $\widehat{G}$ is 1 -transitive it will be equal to the whole of $\operatorname{SO}(2, \mathbb{R})$.

For $x \in \mathbb{S}^{1}$ let $\pi_{x}: \mathbb{R} \rightarrow \mathbb{S}^{1}$ be the usual projection map which sends each integer to $x$ and for each integer translation $T: \mathbb{R} \rightarrow \mathbb{R}$ satisfies $\pi_{x} \circ T=\pi_{x}$.

If we fix $x \in \mathbb{S}^{1}$ then since $G$ is continuously 1-transitive we can choose a continuous path $g:[0,1] \rightarrow G$ such that $g(t)(x)=\pi_{x}(t)$ and $g(0)=$ id. Notice that this path is contained in $\widehat{G}$ and $g(1)$ is not necessarily the identity even though it fixes $x$.

For $x \in \mathbb{S}^{1}$ we define a continuous map $F_{x}: \mathbb{R} \rightarrow \widehat{G}$ by

$$
F_{x}(t)=g(t-[t]) \circ g(1)^{[t]}
$$

where $[t]$ is the greatest integer less than or equal to $t$. Set $f=F_{x}(1)$. Note that $F_{x}(n)=f^{n}$ for every $n \in \mathbb{Z}$.

We claim that $F_{x}$ has the following properties,

(1) $F_{x}(t)(x)=\pi_{x}(t)$ for every $t \in \mathbb{R}$

(2) $F_{x}(0)=\mathrm{id}$

(3) The map $F_{x}$ is a surjection, that is $F_{x}(\mathbb{R})=\widehat{G}$

(4) If the map $f=F_{x}(1)$ is not equal to the identity map then $F_{x}$ is a bijection

The first two properties follow directly from the definition. To see that the third property holds, let $h_{s}$ be a path in $\widehat{G}, s \geq 0, h_{0}=$ id. Let $\alpha(s)=h_{s}(x)$. We have that $\alpha$ is a continuous map from the non-negative reals $\mathbb{R}^{+}$into the circle. Since the set $\mathbb{R}^{+}$ 
is contractible we can lift the map $\alpha$ into the universal cover of the circle. That is, there is a map $\beta: \mathbb{R}^{+} \rightarrow \mathbb{R}$ such that $\pi_{x} \circ \beta=\alpha$. We have $F_{x}(\beta(s))(x)=h_{s}(x)$. Then $\left(h_{s}{ }^{-1} \circ F_{x}(\beta(s))\right)(x)=x$. It follows from the assumption of the theorem that $F_{x}(\beta(s))=h_{s}$ and $F_{x}$ is surjective. The map $F_{x}$ is injective for $0 \leq t<1$, because $F_{x}(t)(x)=\pi_{x}(t)$. If $F_{x}(1)$ is not the identity, and since $F_{x}(1)(x)=x$ we have that $F_{x}(m)=F_{x}(n)$ if and only if $m=n$, for every two integers $m, n$. This implies the fourth property.

It follows from $(*)$, and the surjectivity of $F_{x}$, that $\widehat{G}$ is a compact group if and only if the cyclic group generated by $F_{x}(1)=f$ is a compact group. We will prove that $f=\mathrm{id}$.

Assume that $f$ is not the identity map. Since $F_{x}$ is a bijection for each $t \in \mathbb{R}$ there exists a unique $s_{n}(t) \in \mathbb{R}$ such that,

$$
f^{n} \circ F_{x}(t) \circ f^{-n}=F_{x}\left(s_{n}(t)\right) .
$$

This defines a function $s_{n}: \mathbb{R} \rightarrow \mathbb{R}$ which we claim is continuous for each $n$. To see this, fix $n$ and let $t_{m} \in \mathbb{R}$ be a convergent sequence with limit $t^{\prime}$. Since $F_{x}$ is continuous,

$$
f^{n} \circ F_{x}\left(t_{m}\right) \circ f^{-n} \longrightarrow f^{n} \circ F_{x}\left(t^{\prime}\right) \circ f^{-n}
$$

and so $F_{x}\left(s_{n}\left(t_{m}\right)\right) \rightarrow F_{x}\left(s_{n}\left(t^{\prime}\right)\right)$ as $m \rightarrow \infty$.

Now, if $s_{n}\left(t_{m_{k}}\right)$ is a convergent subsequence, with limit $t_{0}$, then using continuity $F_{x}\left(s_{n}\left(t_{m_{k}}\right)\right)$ will converge to $F_{X}\left(t_{0}\right)$. Since $F_{x}$ is a bijection this gives us that $t_{0}=s_{n}\left(t^{\prime}\right)$. Consequently, if the sequence $s_{n}\left(t_{m}\right)$ were bounded, then it would converge to $t^{\prime}$.

Assume now that the sequence $s_{n}\left(t_{m}\right)$ is unbounded and take a divergent subsequence $s_{n}\left(t_{m_{k}}\right)$. Consider the corresponding sequence,

$$
F_{x}\left(s_{n}\left(t_{m_{k}}\right)\right)=g\left(s_{n}\left(t_{m_{k}}\right)-\left[s_{n}\left(t_{m_{k}}\right)\right]\right) \circ f^{\left[s_{n}\left(t_{m_{k}}\right)\right]} .
$$

Since $s_{n}\left(t_{m_{k}}\right)-\left[s_{n}\left(t_{m_{k}}\right)\right] \in[0,1)$ for each $m$, there exists a subsequence $t_{m_{k_{l}}}$ of $t_{m_{k}}$ such that $s_{n}\left(t_{m_{k_{l}}}\right)-\left[s_{n}\left(t_{m_{k_{l}}}\right)\right]$ converges to some $t_{0} \in[0,1]$. Now since $g$ is continuous and the sequence $F_{x}\left(s_{n}\left(t_{m}\right)\right)$ converges to a homeomorphism $F_{x}\left(s_{n}\left(t^{\prime}\right)\right)$ we have that $f^{\left[s_{n}\left(t_{m_{k_{l}}}\right)\right]}$ converges to a homeomorphism as $l \rightarrow \infty$. However, as $s_{n}\left(t_{m_{k}}\right)$ is divergent $\left[s_{n}\left(t_{m_{k_{l}}}\right)\right]$ will be divergent too.

Let $S_{f}$ denote the set of fixed points of $f$. Note that $x \in S_{f}$. Since we assume that $f$ is not the identity we have that $\mathbb{S}^{1} \backslash S_{f}$ is non-empty. Let $J$ be a component of $\mathbb{S}^{1} \backslash S_{f}$ and let $a, b \in \mathbb{S}^{1}$ be its endpoints. Since $f$ fixes $J$, and has no fixed points inside $J$ we deduce that on compact subsets of $J$ the sequence $f^{\left[s_{n}\left(t_{m_{k_{l}}}\right)\right]}$ converges 
to one of the endpoints and consequently, can not converge to a homeomorphism. This is a contradiction, so $s_{n}\left(t_{m}\right)$ can not be unbounded and $s_{n}$ is continuous.

Notice that $s_{n}(0)=0$ and if $t \in \mathbb{Z}$ then $F_{x}(t)$ will commute with the $f^{n}$ so we have $s_{n}(m)=m$ for all $m \in \mathbb{Z}$. This yields that $s_{n}([0,1])=[0,1]$ for every $n \in \mathbb{Z}$.

Let $U_{f} \subset \mathbb{S}^{1}$ be the set defined as follows. We say that $y \in U_{f}$ if there exists an open interval $I, y \in I$, such that $\left|f^{n}(I)\right| \rightarrow 0, n \rightarrow \infty$. Here $\left|f^{n}(I)\right|$ denotes the length of the corresponding interval. The set $U_{f}$ is open. We show that $U_{f}$ is non-empty and not equal to $\mathbb{S}^{1}$. As before, let $J$ be a component of $\mathbb{S}^{1} \backslash S_{f}$ and let $a, b \in \mathbb{S}^{1}$ be its endpoints. Since $f$ fixes $J$, and has no fixed points inside $J$ we deduce that on compact subsets of $J$ the sequence $f^{n}$ converges to one of the endpoints, say $a$. This shows that $J \subset U_{f}$. Also, this shows that the point $b$ does not belong to $U_{f}$.

Let $y \in U_{f}$, and let $I$ be the corresponding open interval so that $y \in I$ and $\left|f^{n}(I)\right| \rightarrow 0$, $n \rightarrow \infty$. Set $f^{n}(I)=I_{n}$. Consider the interval $F_{x}\left(s_{n}(t)\right)\left(I_{n}\right), t \in[0,1]$. Since $s_{n}([0,1])=[0,1]$ we have that $F_{x}\left(s_{n}([0,1])\right)$ is a compact family of homeomorphisms. This allows us to conclude that $\left|F_{x}\left(s_{n}(t)\right)\left(I_{n}\right)\right| \rightarrow 0, n \rightarrow \infty$, uniformly in $n$ and $t \in[0,1]$. Set $J_{t}=F_{x}(t)(I)$. From $(* *)$ we have that $\left|f^{n}\left(J_{t}\right)\right| \rightarrow 0, n \rightarrow \infty$, for a fixed $t \in[0,1]$. This implies that the point $F_{x}(t)(y)$ belongs to the set $U_{f}$ for every $t \in[0,1]$.

Let $J$ be a component of $U_{f}$, and let $a, b$ be its endpoints. Note that the points $a, b$ do not belong to $U_{f}$. Since $F_{x}(t)$ is a continuous path and $F_{x}(0)=\mathrm{id}$, for small enough $t$ we have that $F_{x}(t)(J) \cap J \neq \varnothing$. Since $F_{x}(t)(J) \subset U_{f}$, and since $a, b$ are not in $U_{f}$ we have that $F_{x}(t)(J)=J$. By continuity this extends to hold for every $t \in[0,1]$. But this means that $F_{x}(t)(a)=a$ for every $t \in[0,1]$. However, for appropriately chosen inverse $t_{0}=\pi_{x}^{-1}(a)$, we have that $F_{x}\left(t_{0}\right)(x)=a$, which contradicts the fact that $F_{x}\left(t_{0}\right)$ is a homeomorphism. This shows that $f=\mathrm{id}$, and therefore we have proved that $\widehat{G}$ is a compact group.

To finish the argument, it remains to show that $G=\widehat{G}$. Let $\Phi \in \operatorname{Homeo}\left(\mathbb{S}^{1}\right)$ be a map which conjugates $\widehat{G}$ to $\operatorname{SO}(2, \mathbb{R})$ and take $g \in G \backslash \widehat{G}$. Since $\widehat{G}$ is a normal subgroup of $G, \Phi \circ g \circ \Phi^{-1}$ conjugates rotations to rotations. Lifting to the universal cover we get that every lift of $\Phi \circ g \circ \Phi^{-1}$ conjugates translations to translations. If we choose one then by Lemma 3.9 it will be affine. On the other hand, it must be periodic, and hence is a translation. So that $\Phi \circ g \circ \Phi^{-1}$ is itself a rotation and we are done.

Theorem 3.10 If $J_{x} \neq \varnothing$ then one of the following is true:

(1) $J_{x}=\mathbb{S}^{1} \backslash\{x\}$ in which case $G$ is continuously 2-transitive. 
(2) There exists $R \in \operatorname{Homeo}\left(\mathbb{S}^{1}\right)$ which is conjugate to a finite order rotation and satisfies $R \circ g=g \circ R$ for every $g \in G$. Moreover, $G$ is a cyclic cover of a group $G_{\Gamma}$ which is continuously 2-transitive, where the covering transformations are the cyclic group generated by $R$.

Proof If $J_{x}=\mathbb{S}^{1} \backslash\{x\}$ then we are in case 4 of Lemma 2.5 with $n=2$. In this situation we know that $G$ will be continuously 2 -transitive.

We already know that $S_{x}=\mathbb{S}^{1} \backslash J_{x}$ must contain $x$ and by Lemma 3.4 must be finite. Moreover, as $f\left(J_{x}\right)=J_{f(x)}$ the sets $S_{x}$ contain the same number of points for each $x \in \mathbb{S}^{1}$. Define $R: \mathbb{S}^{1} \rightarrow \mathbb{S}^{1}$ by taking $R(x)$ to be the first point of $S_{x}$ you come to as you travel anticlockwise around $\mathbb{S}^{1}$. Now take $g \in G$ and $x \in \mathbb{S}^{1}$, then since $J_{g(x)}=g\left(J_{x}\right)$ and $g$ is orientation preserving $R \circ g(x)=g \circ R(x)$ for all $x \in \mathbb{S}^{1}$.

We now show that $R$ is a homeomorphism. To see this take any continuous path $x_{t} \in \mathbb{S}^{1}$, we will show that $R\left(x_{t}\right) \rightarrow R\left(x_{0}\right)$ as $t \rightarrow 0$. Since $G$ is continuously 1 -transitive, there exists a continuous path $g_{t} \in G$ satisfying $g_{t}\left(x_{t}\right)=x_{0}$, so that,

$$
\lim _{t \rightarrow 0} R\left(x_{t}\right)=\lim _{t \rightarrow 0}\left(g_{t}\right)^{-1}\left(R\left(g_{t}\left(x_{t}\right)\right)\right)=\lim _{t \rightarrow 0}\left(g_{t}\right)^{-1}\left(R\left(x_{0}\right)\right)=R\left(x_{0}\right) .
$$

where the first equality follows from the fact that $R \circ g(x)=g \circ R(x)$ for all $x \in \mathbb{S}^{1}$. This shows that $R$ is continuous. If we take $y \notin J_{x}$ then $J_{x} \subset J_{y}$, and hence $S_{x} \supset S_{y}$ but in this case since $S_{x}$ and $S_{y}$ contain the same number of points they will be equal. Consequently, $R$ has an inverse defined by taking $R^{-1}(x)$ to be the first point of $S_{x}$ you come to by traveling clockwise around $\mathbb{S}^{1}$ and this inverse is continuous by the same argument as for $R$. Consequently, $R \in \operatorname{Homeo}\left(\mathbb{S}^{1}\right)$. Furthermore, $R$ is of finite order equal to the number of points in $S_{x}$ and hence conjugate to a rotation.

Let $\Gamma$ denote the cyclic subgroup of Homeo $\left(\mathbb{S}^{1}\right)$ generated by $R$. Define $\pi: \mathbb{S}^{1} \rightarrow$ $\mathbb{S}^{1} / \Gamma \cong \mathbb{S}^{1}$, in the usual way with $\pi(x)$ being the orbit of $x$ under $\Gamma$. Since $R \circ g(x)=g \circ R(x)$ for all $x \in \mathbb{S}^{1}$, each $g \in G$ defines a well defined homeomorphism of the quotient space $\mathbb{S}^{1} / \Gamma$ which we call $g_{\Gamma}$. This gives us a homomorphism $\pi_{\Gamma}: G \rightarrow \operatorname{Homeo}\left(\mathbb{S}^{1}\right)$, defined by $\pi_{\Gamma}(g)=g_{\Gamma}$. Let $G_{\Gamma}$ denote the image of $G$ under $\pi_{\Gamma}$, then $G$ is a cyclic cover of $G_{\Gamma}$.

It remains to see that $G_{\Gamma}$ is continuously 2 -transitive. This follows from the fact that if we take $x_{0} \in \mathbb{S}^{1}$ then $J_{\pi\left(x_{0}\right)}=\pi\left(J_{x_{0}}\right)$, where $J_{\pi\left(x_{0}\right)}$ is the set of points that can be moved by continuous deformations of the identity in $G_{\Gamma}$ which fix $\pi\left(x_{0}\right)$. Consequently, $J_{\pi\left(x_{0}\right)}=\mathbb{S}^{1} \backslash\left\{x_{0}\right\}$ so that $G_{\Gamma}$ is continuously 2-transitive by the first part of this proposition. 


\section{Implications of continuous 2-transitivity}

We now know that if $G$ is transitive and contains a continuous deformation of the identity then it is either conjugate to the group of rotations $\mathrm{SO}(2, \mathbb{R})$, is continuously 2 -transitive, or is a cyclic cover of a group which is continuously 2-transitive. For the rest of the paper we assume that $G$ is continuously 2 -transitive and examine which possibilities arise.

For $n \geq 2$ and $\left(x_{1} \ldots x_{n}\right) \in P_{n}$ we define $J_{x_{1} \ldots x_{n}}$ to be the subset of $\mathbb{S}^{1}$ containing the points $x \in \mathbb{S}^{1}$ which satisfy the following condition. There exists a continuous deformation of the identity $f_{t} \in G$, with $f_{t}\left(x_{i}\right)=x_{i}$ for each $i$ and $t$ and such that there exists $t_{0} \in[0,1]$ with $f_{t_{0}}(x) \neq x$. This generalizes the earlier definition of $J_{x}$ and we get the following analogous results.

Lemma 4.1 $J_{f\left(x_{1}\right) \ldots f\left(x_{n}\right)}=f\left(J_{x_{1} \ldots x_{n}}\right)$ for every $f \in G$.

Lemma 4.2 $J_{x_{1} \ldots x_{n}}$ is open.

We also have the following.

Lemma 4.3 If $J_{x_{1} \ldots x_{n}}$ is nonempty and $G$ is continuously $n$-transitive, then it is equal to $\mathbb{S}^{1} \backslash\left\{x_{1} \ldots x_{n}\right\}$.

Proof Assume that $J_{x_{1} \ldots x_{n}} \subset \mathbb{S}^{1} \backslash\left\{x_{1}, \ldots, x_{n}\right\}$ is nonempty. By Lemma 4.2 it is also open and hence is a countable union of open intervals. Pick one of these, and call its endpoints $b_{1}$ and $b_{2}$. Assume for contradiction that at least one of $b_{1}$ and $b_{2}$ is not one of the $x_{i}$. Interchanging $b_{1}$ and $b_{2}$ if necessary we can assume that this point is $b_{1}$. Since $G$ is continuously $n$-transitive there exist elements of $G$ which cyclically permute the $x_{i}$. Using these elements and the fact that $J_{f\left(x_{1}\right) \ldots f\left(x_{n}\right)}=f\left(J_{x_{1} \ldots x_{n}}\right)$ for every $f \in G$, we can assume without loss of generality that $b_{1}$ and hence the whole interval lies in the component of $\mathbb{S}^{1} \backslash\left\{x_{1}, \ldots, x_{n}\right\}$ whose endpoints are $x_{1}$ and $x_{2}$.

We now claim that $J_{b_{1}, b_{2}, x_{3}, \ldots, x_{n}} \supset J_{x_{1} \ldots x_{n}}$. To see this, take $x \in J_{x_{1} \ldots x_{n}}$, then there exists a continuous deformation of the identity $f_{t}$ which fixes $x_{1}, \ldots, x_{n}$ and for which there exists $t_{0}$ such that $f_{t_{0}}(x) \neq x$. Now since $b_{1}, b_{2} \notin J_{x_{1} \ldots x_{n}}, f_{t}$ must also fix $b_{1}$ and $b_{2}$ for all $t$, consequently we can use $f_{t}$ to show that $x \in J_{b_{1}, b_{2}, x_{3}, \ldots, x_{n}}$. In particular, this means that $J_{b_{1}, b_{2}, x_{3}, \ldots, x_{n}}$ contains the whole interval between $b_{1}$ and $b_{2}$.

Take $g \in G$ which maps $\left\{b_{1}, b_{2}\right\}$ to $\left\{x_{1}, x_{2}\right\}$ and fixes the other $x_{i}$, such an element exists as $G$ is continuously $n$-transitive. Then,

$$
J_{x_{1}, x_{2}, x_{3}, \ldots, x_{n}}=J_{g\left(b_{1}\right), g\left(b_{2}\right), g\left(x_{3}\right), \ldots, g\left(x_{n}\right)}=g\left(J_{b_{1}, b_{2}, x_{3}, \ldots, x_{n}}\right)
$$


so that $J_{x_{1} \ldots x_{n}}$ must contain the whole interval between $x_{1}$ and $x_{2}$. This is a contradiction, since $b_{1}$ lies between $x_{1}$ and $x_{2}$ but is not in $J_{x_{1} \ldots x_{n}}$.

Proposition 4.4 Let $G$ be continuously $n$-transitive for some $n \geq 2$ and suppose there exist $n$ distinct points $a_{1}, \ldots, a_{n} \in \mathbb{S}^{1}$ and a continuous deformation of the identity $g_{t} \in G$, which fixes each $a_{i}$ for all $t$. Then $G$ is continuously $n+1$ transitive.

Proof $J_{a_{1} \ldots a_{n}} \neq \varnothing$ so by Lemma $4.3 J_{a_{1} \ldots a_{n}}=\mathbb{S}^{1} \backslash\left\{a_{1}, \ldots, a_{n}\right\}$. We can now apply Lemma 2.5 to see that $G$ is continuously $n+1$-transitive.

Corollary 4.5 If $G$ is continuously 2-transitive and there exists $g \in G \backslash\{\mathrm{id}\}$ with an open interval $I \subset \mathbb{S}^{1}$ such that the restriction of $g$ to $I$ is the identity, then $G$ is continuously $n$-transitive for every $n \geq 2$.

Proof Let $I \subset \mathbb{S}^{1}$ be a maximal interval on which $g$ acts as the identity, so that if $I^{\prime} \supset I$ is another interval containing $I$ then $g$ doesn't act as the identity on $I^{\prime}$. Let $a$ and $b$ be the endpoints of $I$ and let $a_{t}$ and $b_{t}$ be continuous injective paths with $a_{0}=a, b_{0}=b$ and $a_{t}, b_{t} \notin I$ for each $t \neq 0$. This is possible because $g \neq$ id so that $\mathbb{S}^{1} \backslash I$ will be a closed interval containing more than one point. Let $g_{t}$ be a continuous path in $G$ so that $g_{0}=\mathrm{id}, g_{t}(a)=a_{t}$ and $g_{t}(b)=b_{t}$, such a path exists as $G$ is continuously 2 -transitive.

Consider the path $h_{t}=g^{-1} \circ g_{t} \circ g \circ g_{t}^{-1}$ since $g_{0}=\mathrm{id}$ we get $h_{0}=\mathrm{id}$. Now $g_{t} \circ g \circ g_{t}^{-1}$ acts as the identity on the interval between $a_{t}$ and $b_{t}$ and by maximality of $I, g^{-1}$ will not act as the identity for $t \neq 0$. Consequently, $h_{t}$ is a continuous deformation of the identity which acts as the identity on $I$. So if $G$ is continuously $k$-transitive for $k \geq 2$, by taking $k$-points in $I$ and using Proposition 4.4 we get that $G$ is $k+1$-transitive. As a result, since $G$ is continuously 2 -transitive it will be $n$-transitive for every $n \geq 2$.

$\operatorname{SO}(2, \mathbb{R})$ is an example of a subgroup of $\operatorname{Homeo}\left(\mathbb{S}^{1}\right)$ which is continuously 1 -transitive but not continuously 2-transitive. However, as the next result shows, there are no subgroups of Homeo $\left(\mathbb{S}^{1}\right)$ which are continuously 2 -transitive but not continuously 3-transitive.

Proposition 4.6 If $G$ is continuously 2-transitive, then it is continuously 3-transitive.

Proof Let $a, b \in \mathbb{S}^{1}$ be distinct points. Construct two injective paths $a(t), b(t)$ in $\mathbb{S}^{1}$ with disjoint images, such that $a(0)=a, b(0)=b$ and such that $a(t)$ and $b(t)$ lie in 
the same component of $\mathbb{S}^{1} \backslash\{a, b\}$ for $t \in(0,1]$. We label this component $I$ and the other $I^{\prime}$.

Since $G$ is continuously 2-transitive, there exists a path $g(t) \in G$ such that $g(0)=\mathrm{id}$, $g(t)(a)=a(t)$ and $g(t)(b)=b(t)$ for every $t$. Now for every $t$ the restriction of $g(t)$ to the closure of $I$, is a continuous map of a closed interval into itself, and hence must have a fixed point, $c(t)$. This point will normally not be unique, but since $g(t)$ is continuous, for a small enough time interval we can choose it to depend continuously on $t$. Likewise for the restriction of $g(t)^{-1}$ to the closure of $I^{\prime}$, for a small enough time interval we can choose a path of fixed points $d(t)$, which must therefore also be fixed points for $g(t)$.

Now pick points $c \in I$ and $d \in I^{\prime}$. Using continuous 2-transitivity of $G$ construct a path $h(t) \in G$ such that $h(t)(c)=c(t)$ and $h(t)(d)=d(t)$. Then $h_{t}^{-1} \circ g(t) \circ h_{t}$ is only the identity when $t=0$ because the same is true of $g(t)$ and we have constructed a continuous deformation of the identity which fixes $c$ and $d$ for all $t$. Consequently we can use Proposition 4.4 to show that $G$ is continuously 3-transitive.

\section{Convergence Groups}

Definition 5.1 A subgroup $G$ of Homeo( $\left.\mathbb{S}^{1}\right)$ is a convergence group if for every sequence of distinct elements $g_{n} \in G$, there exists a subsequence $g_{n_{k}}$ satisfying one of the following two properties:

(1) There exists $g \in G$ such that,

$$
\lim _{k \rightarrow \infty} g_{n_{k}}=g \text { and } \lim _{k \rightarrow \infty} g_{n_{k}}^{-1}=g^{-1}
$$

uniformly in $\mathbb{S}^{1}$.

(2) There exist points $x_{0}, y_{0} \in \mathbb{S}^{1}$ such that,

$$
\lim _{k \rightarrow \infty} g_{n_{k}}=x_{0} \text { and } \lim _{k \rightarrow \infty} g_{n_{k}}^{-1}=y_{0}
$$

uniformly on compact subsets of $\mathbb{S}^{1} \backslash\left\{y_{0}\right\}$ and $\mathbb{S}^{1} \backslash\left\{x_{0}\right\}$ respectively.

The notion of convergence groups was introduced by Gehring and Martin [4] and they have proceeded to play a central role in geometric group theory. The following theorem has been one of the most important and we shall make frequent use of it.

Theorem 5.2 $G$ is a convergence group if and only if it is conjugate in Homeo( $\left.\mathbb{S}^{1}\right)$ to a subgroup of PSL $(2, \mathbb{R})$. 
This Theorem was proved by Gabai in [3]. Prior to that, Tukia [7] proved this result in many cases and Hinkkanen [6] proved it for non discrete groups. Casson and Jungreis proved it independently using different methods [2]. See [2], [3], [7] for references to other papers in this subject.

For the rest of this section we shall assume that $G$ is continuously $n$-transitive, but not continuously $n+1$-transitive for some $n \geq 3$.

Take $\left(x_{1}, \ldots, x_{n-1}\right) \in P_{n-1}$ and define

$$
G_{0}=\left\{g \in G: g\left(x_{i}\right)=x_{i} \quad i=1, \ldots, n-1\right\} .
$$

Choose a component $I$ of $\mathbb{S}^{1} \backslash\left\{x_{1}, \ldots, x_{n-1}\right\}$ and denote its closure by $\bar{I}$. We construct a homomorphism $\Phi: G_{0} \rightarrow \operatorname{Homeo}\left(\mathbb{S}^{1}\right)$ as follows. Take $g \in G_{0}$, then since $g$ fixes the endpoints of $I$ and is orientation preserving, we can restrict it to a homeomorphism $g^{\prime}$ of $\bar{I}$. By identifying the endpoints of $\bar{I}$ we get a copy of $\mathbb{S}^{1}$ and we define $\Phi(g)$ to be the homeomorphism of $\mathbb{S}^{1}$ that $g^{\prime}$ descends to under this identification. We label the identification point $\bar{x}$ and set $\mathcal{G}_{0}=\Phi\left(G_{0}\right)$ to be the image of $G_{0}$ under $\Phi$.

In this situation Lemma 2.5 implies the following. For every $x \in I$, there exists a continuous map $F_{x}: \mathbb{S}^{1} \backslash \bar{x} \rightarrow \mathcal{G}_{0}$ satisfying the properties,

(1) $\left(F_{x}(y)\right)(x)=y \quad \forall y \in \mathbb{S}^{1} \backslash \bar{x}$

(2) $F_{x}(x)=\mathrm{id}$.

Proposition 5.3 $\Phi: G_{0} \rightarrow \mathcal{G}_{0}$ is an isomorphism.

Proof Surjectivity is trivial. If we assume that $\Phi$ is not injective then there will exist $g \in G_{0}$ which is non-trivial and acts as the identity on $I$. Then by Corollary $4.5 G$ will be $n+1$ transitive, a contradiction.

Let $\widehat{G}_{0}$ denote the path component of the identity in $G_{0}$, we now analyze the group $\widehat{\mathcal{G}}_{0}=\Phi\left(\widehat{G}_{0}\right)$.

Proposition $5.4 \widehat{\mathcal{G}}_{0}$ is a convergence group.

Proof Choose $x \in I$ then we know there exists a continuous map $F_{x}: \mathbb{S}^{1} \backslash \bar{x} \rightarrow \mathcal{G}_{0}$ satisfying the properties,

(1) $\left(F_{x}(y)\right)(x)=y \quad \forall y \in \mathbb{S}^{1} \backslash \bar{x}$

(2) $F_{x}(x)=\mathrm{id}$. 
Now since $F_{x}(x)=$ id and $F_{X}$ is continuous, the image of $F_{x}$ will lie entirely in $\widehat{\mathcal{G}}_{0}$. In fact, $F_{x}$ gives a bijection between $\mathbb{S}^{1} \backslash \bar{x}$ and $\widehat{\mathcal{G}}_{0}$. To see this we first observe that injectivity follows directly from condition 1 . To see that it is also surjective, take $g \in \widehat{\mathcal{G}}_{0}$. Then there exists a path $g_{t} \in \widehat{\mathcal{G}}_{0}$ for $t \in[0,1]$ with $g_{0}=$ id and $g_{1}=g$. So that $g_{t}(x)$ is a path in $\mathbb{S}^{1} \backslash \bar{x}$ from $x$ to $g(x)$. Consider the path $\left(F_{x}\left(g_{t}(x)\right)\right)^{-1} \circ g_{t}$ in $\widehat{\mathcal{G}}_{0}$, it fixes $x$ for every $t$, and so must be the identity for each $t$. Otherwise, by Proposition 4.4, $G$ would be continuously $n+1$-transitive, which would contradict our assumptions. As a result $g=F_{x}(g(x))$ so $F_{x}$ is a bijection, with inverse given by evaluation at $x$.

Fix $x_{0} \in \mathbb{S}^{1} \backslash \bar{x}$, let $g_{n}$ be a sequence of elements of $\widehat{\mathcal{G}}_{0}$ and consider the sequence of points $g_{n}\left(x_{0}\right)$, since $\mathbb{S}^{1}$ is compact $g_{n}\left(x_{0}\right)$ has a convergent subsequence $g_{n_{k}}\left(x_{0}\right)$ converging to some point $x^{\prime}$. If $x^{\prime} \neq \bar{x}$ then by continuity of $F_{x_{0}}, g_{n_{k}}$ will converge to $F_{x_{0}}\left(x^{\prime}\right)$. Now if there does not exist a subsequence of $g_{n}\left(x_{0}\right)$ converging to some $x^{\prime} \neq \bar{x}$, then take a subsequence $g_{n_{k}}$ such that $g_{n_{k}}\left(x_{0}\right)$ converges to $\bar{x}$. If we can show that $g_{n_{k}}(x)$ converges to $\bar{x}$ for every $x \in \mathbb{S}^{1} \backslash \bar{x}$ then we shall be done.

Suppose for contradiction that there exists $x \in \mathbb{S}^{1} \backslash \bar{x}$ such that $g_{n_{k}}(x)$ does not converge to $\bar{x}$. Then there exists a subsequence of $g_{n_{k}}(x)$ which converges to $x^{\prime} \neq \bar{x}$, but then by the previous argument the corresponding subsequence of $g_{n_{k}}$ will converge to the homeomorphism $F_{x}\left(x^{\prime}\right)$. This is a contradiction since $F_{x}\left(x^{\prime}\right)\left(x_{0}\right)$ would have to equal $\bar{x}$.

Corollary 5.5 Let $g$ be an element of $\widehat{\mathcal{G}}_{0}$. If $g$ fixes a point in $\mathbb{S}^{1} \backslash \bar{x}$ then it is the identity.

Proof Let $x \in \mathbb{S}^{1} \backslash \bar{x}$ be a fixed point of $g$. From the previous proof we know that $F_{x}: I \rightarrow \widehat{\mathcal{G}}_{0}$ is a bijection. So that $F_{x}(g(x))=g$, but $g$ fixes $x$ so that $g=F_{x}(x)=$ id.

Corollary 5.6 The restriction of the action of $\widehat{\mathcal{G}}_{0}$ to $\mathbb{S}^{1} \backslash \bar{x}$ is conjugate to the action of $\mathbb{R}$ on itself by translation.

Proof By Theorem 5.2 and Proposition $5.4 \widehat{\mathcal{G}}_{0}$ is conjugate in Homeo( $\left.\mathbb{S}^{1}\right)$ to a subgroup of $\operatorname{PSL}(2, \mathbb{R})$ which fixes the point $\bar{x}$. Moreover, from Corollary 5.5 this is the only point fixed by a non trivial element. By identifying $\mathbb{S}^{1}$ with $\mathbb{R} \cup\{\infty\}$ so that $\bar{x}$ is identified with $\{\infty\}$ in the usual way, we see that $\widehat{\mathcal{G}}_{0}$ is conjugate to a subgroup of the Möbius group acting on $\mathbb{R} \cup\{\infty\}$. Since every element will fix $\{\infty\}$, their restriction to $\mathbb{R}$ will be an element of $\operatorname{Aff}(\mathbb{R})$ acting without fixed points, so can only be a translation. On the other hand the group must act transitively on $\mathbb{R}$ and so must be the full group of translations. This gives the result. 
Proposition 5.7 The restriction of the action of $G_{0}$ to $I$ is conjugate to the action of a subgroup of the affine group $\mathrm{Aff}(\mathbb{R})$ on $\mathbb{R}$. In particular, each non trivial element of $G_{0}$ can act on $I$ with at most one fixed point.

Proof The restriction of $\widehat{\mathcal{G}}_{0}$ to $\mathbb{S}^{1} \backslash \bar{x}$ is isomorphic to the restriction of $\widehat{G}_{0}$ to $I$. So that by Corollary 5.6 there exists a homeomorphism $\phi: I \rightarrow \mathbb{R}$ which conjugates the restriction of $\widehat{G}_{0}$ to $I$, to the action of $\mathbb{R}$ on itself by translation. Take $h \in G_{0} \backslash \widehat{G}_{0}$ then $h^{\prime}=\phi \circ h \circ \phi^{-1}$ is a self-homeomorphism of $\mathbb{R}$. Since $\widehat{G}_{0}$ is a normal subgroup of $G_{0}, h^{\prime}$ conjugates every translation to another one and so by Lemma 3.9 is itself an affine map and the proof is complete.

Let $g$ be a nontrivial element of $G_{0}$, then $g \in \widehat{G}_{0}$ if and only if it acts on each component of $\mathbb{S}^{1} \backslash\left\{x_{1}, \ldots, x_{n-1}\right\}$ as a conjugate of a non trivial translation. Furthermore, if $g \notin \widehat{G}_{0}$ then it acts on each component of $\mathbb{S}^{1} \backslash\left\{x_{1}, \ldots, x_{n-1}\right\}$ as a conjugate of a affine map which is not a translation, each of which must have a fixed point. This situation cannot actually arise as the next proposition will show.

Proposition 5.8 $G_{0}=\widehat{G}_{0}$

Proof Let $g \in G_{0} \backslash \widehat{G}_{0}$, then $g$ acts on each component of $\mathbb{S}^{1} \backslash\left\{x_{1}, \ldots, x_{n-1}\right\}$ as a conjugate of a affine map which is not a translation. Consequently, $g$ will have a fixed point in each component of $\mathbb{S}^{1} \backslash\left\{x_{1}, \ldots, x_{n-1}\right\}$. Label the fixed points of $g$ in the components of $\mathbb{S}^{1} \backslash\left\{x_{1}, \ldots, x_{n-1}\right\}$ whose boundaries both contain $x_{1}$ as $y_{1}$ and $y_{2}$. Since $G$ is $n$-transitive, there exists a map $g^{\prime}$ which sends $y_{1}$ to $x_{1}$ and fixes all the other $x_{i}$. Then $g^{\prime} \circ g \circ\left(g^{\prime}\right)^{-1}$ fixes all the $x_{i}$ and hence is an element of $G_{0}$. On the other hand, $g^{\prime} \circ g \circ\left(g^{\prime}\right)^{-1}$ also fixes $g^{\prime}\left(x_{1}\right)$ and $g^{\prime}\left(y_{2}\right)$ which lie in the same component of $\mathbb{S}^{1} \backslash\left\{x_{1}, \ldots, x_{n-1}\right\}$, this is impossible since every non-trivial element of $G_{0}$ can only have one fixed point in each component of $\mathbb{S}^{1} \backslash\left\{x_{1}, \ldots, x_{n-1}\right\}$.

Corollary 5.9 The restriction of the action of $G_{0}$ to $I$ is conjugate to the action of $\mathbb{R}$ on itself by translation. In particular the action is free.

We finish this section by comparing the directions that a non-trivial element of $G_{0}$ moves points in different components of $\mathbb{S}^{1} \backslash\left\{x_{1}, \ldots, x_{n-1}\right\}$. So endow $\mathbb{S}^{1}$ with the anti-clockwise orientation, this gives us an ordering on any interval $I \subset \mathbb{S}^{1}$, where for distinct points $x, y \in I, x \prec y$ if one travels in an anti-clockwise direction to get from $x$ to $y$ in $I$. Let $g \in G_{0} \backslash\{$ id $\}$ if $I$ is a component of $\mathbb{S}^{1} \backslash\left\{x_{1}, \ldots, x_{n-1}\right\}$ then we shall say that $g$ acts positively on $I$ if $x \prec g(x)$ and negatively if $x \succ g(x)$ for one and hence every $x \in I$. 
Let $I$ and $I^{\prime}$ be the two components of $\mathbb{S}^{1} \backslash\left\{x_{1}, \ldots, x_{n-1}\right\}$ whose boundaries contain $x_{i}$. Labeled so that in the order on the closure of $I, x \prec x_{i}$ for each $x \in I$, whereas in the order on the closure of $I^{\prime}, x_{i} \prec x$ for each $x \in I^{\prime}$. Then we have the following,

Proposition 5.10 Let $g$ be a non trivial element of $G_{0}$, if $g$ acts positively on $I$ then it acts negatively on $I^{\prime}$ and if $g$ acts negatively on $I$ then it acts positively on $I^{\prime}$.

Proof Let $x, x^{\prime} \in I$ and $y, y^{\prime} \in I^{\prime}$ be points such that $x \prec x^{\prime}$ and $y \succ y^{\prime}$. There exists $g \in G$ fixing $x_{1}, \ldots, x_{i-1}$ and $x_{i+1}, \ldots, x_{n-1}$ and sending $x$ to $x^{\prime}$ and $y$ to $y^{\prime}$. This map will have a fixed point $\tilde{x}$ between $x^{\prime}$ and $y^{\prime}$, since it maps the interval between them into itself.

Let $g^{\prime} \in G$ fix $x_{1}, \ldots, x_{i-1}$ and $x_{i+1}, \ldots, x_{n-1}$ and send $\tilde{x}$ to $x_{i}$. Then $g_{0}=$ $g^{\prime} \circ g \circ\left(g^{\prime}\right)^{-1}$ will fix $x_{1}, \ldots, x_{n-1}$ and hence lie in $G_{0}$. Moreover, $g_{0}$ acts positively on $I$ and negatively on $I^{\prime}$.

Now let $g_{1} \in G_{0}$ be any non-trivial element which acts positively on $I$. Then there exists a path $g_{t}$ in $G_{0}$ from $g_{0}=g^{\prime} \circ g \circ\left(g^{\prime}\right)^{-1}$ to $g_{1}$, so that $g_{t} \neq$ id for any $t$. Since $g_{t}$ is never the identity and $g_{0}$ acts negatively on $I^{\prime}, g_{1}$ must also act negatively on $I^{\prime}$.

If $h \in G_{0}$ is a non-trivial element which acts negatively on $I$, then $h^{-1}$ will act positively on $I$. So that, by the above argument, $h^{-1}$ will act negatively on $I^{\prime}$. This means that $h$ will act positively on $I^{\prime}$ as required.

Corollary 5.11 If $G$ is $n$-transitive but not $n+1$-transitive for $n \geq 3$ then $n$ is odd.

Proof Let $g$ be a non-trivial element of $G_{0}$ which acts positively on some component $I$ of $\mathbb{S}^{1} \backslash\left\{x_{1}, \ldots, x_{n-1}\right\}$. Then by Proposition 5.10 as we travel around $\mathbb{S}^{1}$ in an anti-clockwise direction the manner in which it acts on each component will alternate between negative and positive. Consequently, if $n$ was even, when we return to $I$ we would require that $g$ acted negatively on $I$, a contradiction, so $n$ is odd.

\section{Continuous 3-transitivity and beyond}

We begin this section by analyzing the case where $G$ is continuously 3 -transitive but not continuously 4-transitive. We shall show that such a group is a convergence group and consequently conjugate to a subgroup of $\operatorname{PSL}(2, \mathbb{R})$.

Fix distinct points $x_{0}, y_{0} \in \mathbb{S}^{1}$ and define

$$
G_{0}=\left\{g \in G: g\left(x_{0}\right)=x_{0}, g\left(y_{0}\right)=y_{0}\right\}
$$




$$
\bar{G}=\left\{g \in G: g\left(x_{0}\right)=x_{0}\right\}
$$

then we have the following propositions.

Proposition 6.1 $G_{0}$ is a convergence group.

Proof From Corollary 5.9, we know that the restriction of $G_{0}$ to each of the components of $\mathbb{S}^{1} \backslash\left\{x_{0}, y_{0}\right\}$ is conjugate to the action of $\mathbb{R}$ on itself by translation. Let $g_{n}$ be a sequence of distinct elements of $G_{0}$ and take a point $x \in \mathbb{S}^{1} \backslash\left\{x_{0}, y_{0}\right\}$. Then the sequence of points $g_{n}(x)$ will have a convergent subsequence $g_{n_{k}}(x)$. If this sequence converges to $x_{0}$ or $y_{0}$, then from Proposition 5.10 so will the sequences $g_{n_{k}}(y)$ for all $y \in \mathbb{S}^{1} \backslash\left\{y_{0}\right\}$ or $\mathbb{S}^{1} \backslash\left\{x_{0}\right\}$ respectively.

Let $I_{x}$ be the component of $\mathbb{S}^{1} \backslash\left\{x_{0}, y_{0}\right\}$ containing $x$. Assume that the sequence of points $g_{n_{k}}(x)$ converges to a point $x^{\prime} \in I_{x}$. Now let $y$ be a point in the other component, $I_{y}$ of $\mathbb{S}^{1} \backslash\left\{x_{0}, y_{0}\right\}$, and consider the sequence of points $g_{n_{k}}(y)$ in $I_{y}$. If it had a subsequence which converged to $x_{0}$ or $y_{0}$ then the sequence $g_{n_{k}}(x)$ would have to as well. This is impossible so $g_{n_{k}}(y)$ must stay within a compact subset of $I_{y}$ and hence $g_{n_{k}}$ has a subsequence, $g_{n_{k_{l}}}$ for which $g_{n_{k_{l}}}(y)$ converges to some point $y^{\prime} \in I_{y}$.

By Corollary 5.9 there exist self homeomorphisms of $I_{x}$ and $I_{y}$ to which the sequence $g_{n_{k_{l}}}$ converges uniformly on $I_{x}$ and $I_{y}$ respectively. Gluing these together at $x_{0}$ and $y_{0}$ gives us an element of $\operatorname{Homeo}\left(\mathbb{S}^{1}\right)$ which $g_{n_{k}}$ converges to uniformly. Consequently, $G_{0}$ is a convergence group.

Proposition 6.2 $\bar{G}$ is a convergence group.

Proof Let $f_{n}$ be a sequence of elements of $\bar{G}$. If for every $y \in \mathbb{S}^{1} \backslash\left\{x_{0}\right\}$ every convergent subsequence of $f_{n}(y)$ converges to $x_{0}$ then we would be done. So assume that this is not the case, take $y \in \mathbb{S}^{1} \backslash\left\{x_{0}\right\}$ such that the sequence of points $f_{n}(y)$ has a convergent subsequence $f_{n_{k}}(y)$ converging to some point $\tilde{y} \neq x_{0}$. Let $I$ be a small open interval around $\tilde{y}$, not containing $x_{0}$ then since $G$ is continuously 3 -transitive, there exists a map $F_{\tilde{y}}: I \rightarrow \bar{G}$ satisfying the following,

(1) $F_{\widetilde{y}}(x)(\tilde{y})=x$ for all $x \in I$

(2) $F_{\tilde{y}}(\tilde{y})$ is the identity.

Let $g_{1}, g_{2} \in \bar{G}$ satisfy $g_{1}(\tilde{y})=y_{0}$ and $g_{2}\left(y_{0}\right)=y$ consider the sequence,

$$
h_{k}=g_{1} \circ F_{\tilde{y}}\left(f_{n_{k}}(y)\right)^{-1} \circ f_{n_{k}} \circ g_{2}
$$

of elements of $\bar{G}$. They all fix $y_{0}$, and since $g_{1} \circ F_{\widetilde{y}}\left(f_{n_{k}}(y)\right)^{-1}$ converges to $g_{1}$ as $k \rightarrow \infty$ we have the following. 
(1) If $h_{k}$ contains a subsequence $h_{k_{l}}$ such that there exists a homeomorphism $h$ with,

$$
\lim _{l \rightarrow \infty} h_{k_{l}}=h \text { and } \lim _{l \rightarrow \infty}\left(h_{k_{l}}\right)^{-1}=h^{-1}
$$

then so does $f_{n_{k}}$.

(2) Furthermore, if there exist points $x^{\prime}, y^{\prime} \in \mathbb{S}^{1}$ and a subsequence $h_{k_{l}}$ of $h_{k}$ such that,

$$
\lim _{l \rightarrow \infty} h_{k_{l}}=x^{\prime} \text { and } \quad \lim _{l \rightarrow \infty}\left(h_{k_{l}}\right)^{-1}=y^{\prime}
$$

uniformly on compact subsets of $\mathbb{S}^{1} \backslash\left\{y^{\prime}\right\}$ and $\mathbb{S}^{1} \backslash\left\{x^{\prime}\right\}$ respectively, then so does $f_{n_{k}}\left(x^{\prime}\right.$ and $y^{\prime}$ will be replaced by $g_{1}^{-1}\left(x^{\prime}\right)$ and $\left.g_{1}^{-1}\left(y^{\prime}\right)\right)$.

Now, since $G_{0}$ is a convergence group, one of the above situations must occur. Consequently, $\bar{G}=\left\{g \in G: g\left(x_{0}\right)=x_{0}\right\}$ is a convergence group.

Proposition 6.3 If $G$ is a subgroup of Homeo $\left(\mathbb{S}^{1}\right)$ which is continuously 3-transitive but not continuously 4-transitive then $G$ is a convergence group.

Proof This proof is almost identical to the previous one but we write it out in full for clarity.

Choose $x_{0} \in \mathbb{S}^{1}$ and let $f_{n}$ be a sequence of elements of $G$. Then since $\mathbb{S}^{1}$ is compact, the sequence of points $f_{n}\left(x_{0}\right)$ will have a convergent subsequence, $f_{n_{k}}\left(x_{0}\right)$, converging to some point $\tilde{x}$. Let $I$ be a small open interval around $\tilde{x}$, then since $G$ is continuously 3-transitive, there exists a map $F_{\tilde{x}}: I \rightarrow G$ satisfying the following,

(1) $F_{\tilde{x}}(x)(\tilde{x})=x$ for all $x \in I$

(2) $F_{\tilde{x}}(\tilde{x})$ is the identity.

Let $g \in G$ send $\tilde{x}$ to $x_{0}$ and consider the sequence,

$$
h_{k}=g \circ F_{\tilde{x}}\left(f_{n_{k}}\left(x_{0}\right)\right)^{-1} \circ f_{n_{k}}
$$

of elements of $G$. They all fix $x_{0}$, and since $g \circ F_{\tilde{x}}\left(f_{n_{k}}\left(x_{0}\right)\right)^{-1}$ converges to $g$ as $k \rightarrow \infty$ we have the following.

(1) If $h_{k}$ contains a subsequence $h_{k_{l}}$ such that there exists a homeomorphism $h$ with,

$$
\lim _{l \rightarrow \infty} h_{k_{l}}=h \text { and } \lim _{l \rightarrow \infty}\left(h_{k_{l}}\right)^{-1}=h^{-1}
$$

then so does $f_{n_{k}}$. 
(2) Furthermore, if there exist points $x^{\prime}, y^{\prime} \in \mathbb{S}^{1}$ and a subsequence $h_{k_{l}}$ of $h_{k}$ such that,

$$
\lim _{l \rightarrow \infty} h_{k_{l}}=x^{\prime} \text { and } \quad \lim _{l \rightarrow \infty}\left(h_{k_{l}}\right)^{-1}=y^{\prime}
$$

uniformly on compact subsets of $\mathbb{S}^{1} \backslash\left\{y^{\prime}\right\}$ and $\mathbb{S}^{1} \backslash\left\{x^{\prime}\right\}$ respectively, then so does $f_{n_{k}}\left(x^{\prime}\right.$ and $y^{\prime}$ will be replaced by $g^{-1}\left(x^{\prime}\right)$ and $\left.g^{-1}\left(y^{\prime}\right)\right)$.

Now, since $\bar{G}=\left\{g \in G: g\left(x_{0}\right)=x_{0}\right\}$ is a convergence group $G$ is too.

We now look at the case where $G$ is continuously 4-transitive. In this case, we show that $G$ must be $n$-transitive for every $n \in \mathbb{N}$.

Theorem 6.4 If $G$ is continuously $n$-transitive for $n \geq 4$, then it is continuously $n+1$-transitive.

Proof Fix $n \geq 4$ and assume for contradiction that $G$ is continuously $n$-transitive but not continuously $n+1$-transitive. Take $\left(a_{1}, \ldots, a_{n-2}\right) \in P_{n-2}$ and define,

$$
\bar{G}=\left\{g \in G: g\left(a_{i}\right)=a_{i} \forall i\right\}
$$

Let $I$ be a component of $\mathbb{S}^{1} \backslash\left\{a_{1}, \ldots, a_{n-2}\right\}$. Construct a homomorphism $\Psi: \bar{G} \rightarrow$ $\operatorname{Homeo}\left(\mathbb{S}^{1}\right)$ in the same way as $\Phi: G_{0} \rightarrow \operatorname{Homeo}\left(\mathbb{S}^{1}\right)$ was constructed in Section 5. Explicitly, take $g \in \bar{G}$, restrict it to a self homeomorphism of $\bar{I}$ and identify the endpoints to get an element of Homeo $\left(\mathbb{S}^{1}\right)$.

Let $\overline{\mathcal{G}}$ denote the image of $\bar{G}$ under $\Psi$. Then as in Proposition $5.3 \overline{\mathcal{G}}$ is isomorphic to $\bar{G}$. Using the arguments from the earlier Propositions in this section we can show that $\overline{\mathcal{G}}$ is a convergence group and hence conjugate to a subgroup of $\operatorname{PSL}(2, \mathbb{R})$. On the other hand, $\overline{\mathcal{G}}$ is 2 -transitive on $I$ and every element fixes the identification point. This means that the action of $\bar{G}$ on $I$ must be conjugate to the action of $\operatorname{Aff}(\mathbb{R})$ on $\mathbb{R}$.

Let $I$ and $I^{\prime}$ be two components of $\mathbb{S}^{1} \backslash\left\{a_{1}, \ldots, a_{n-2}\right\}$ and let $\phi: I \rightarrow \mathbb{R}$ be a homeomorphism which conjugates the action of $\bar{G}$ on $I$ to the action of $\operatorname{Aff}(\mathbb{R})$ on $\mathbb{R}$. Let $a_{n-1}, a_{n-1}^{\prime}$ be two distinct points in $I^{\prime}$. Consider the groups

$$
G_{0}=\left\{g \in \bar{G}: g\left(a_{n-1}\right)=a_{n-1}\right\}
$$

and

$$
G_{0}^{\prime}=\left\{g \in \bar{G}: g\left(a_{n-1}^{\prime}\right)=a_{n-1}^{\prime}\right\}
$$

They each act transitively on $I$ and by Corollary 5.5 and Proposition 5.8 without fixed points. Consequently, $\phi$ conjugates both of these actions to the action of $\mathbb{R}$ on itself by translation. Let $g \in G_{0}$ and $g^{\prime} \in G_{0}^{\prime}$ be elements which are conjugated to $x \mapsto x+1$ 
by $\phi$. Then $g^{-1} \circ g^{\prime}$ acts on $I$ as the identity. However, if it is equal to the identity, then $g^{\prime}=g$ fixes $a_{n-1}$ and $a_{n-1}^{\prime}$, this is impossible as non-trivial elements of $\bar{G}$ can have at most one fixed point in $I^{\prime}$. So $g^{-1} \circ g$ is a non-trivial element of $G$ which acts as the identity on $I$ and so by Corollary 4.5 we have that $G$ is continuously $n+1$-transitive.

\section{Summary of Results}

Theorem 7.1 Let $G$ be a transitive subgroup of Homeo( $\left(\mathbb{S}^{1}\right)$ which contains a non constant continuous path. Then one of the following mutually exclusive possibilities holds:

(1) $G$ is conjugate to $\mathrm{SO}(2, \mathbb{R})$ in $\operatorname{Homeo}\left(\mathbb{S}^{1}\right)$.

(2) $G$ is conjugate to $\operatorname{PSL}(2, \mathbb{R})$ in $\operatorname{Homeo}\left(\mathbb{S}^{1}\right)$.

(3) For every $f \in \operatorname{Homeo}\left(\mathbb{S}^{1}\right)$ and each finite set of points $x_{1}, \ldots, x_{n} \in \mathbb{S}^{1}$ there exists $g \in G$ such that $g\left(x_{i}\right)=f\left(x_{i}\right)$ for each $i$.

(4) $G$ is a cyclic cover of a conjugate of $\operatorname{PSL}(2, \mathbb{R})$ in Homeo( $\left.\mathbb{S}^{1}\right)$ and hence conjugate to $\operatorname{PSL}_{k}(2, \mathbb{R})$ for some $k>1$.

(5) $G$ is a cyclic cover of a group satisfying condition 3 above.

Proof Let $f:[0,1] \rightarrow G$ be a non constant continuous path. Then

$$
f(0)^{-1} \circ f:[0,1] \rightarrow G
$$

is a continuous deformation of the identity in $G$. Consequently, Proposition 2.6 tells us that $G$ is continuously 1 -transitive.

If $J_{x}=\varnothing$ for every $x \in \mathbb{S}^{1}$ then by Theorem $3.8 G$ is conjugate to $\operatorname{SO}(2, \mathbb{R})$ in Homeo $\left(\mathbb{S}^{1}\right)$. If $J_{x} \neq \varnothing$ for some and hence all $x \in \mathbb{S}^{1}$ then by Theorem $3.10 G$ is either continuously 2 -transitive or is a cyclic cover of a group $G^{\prime}$ which is continuously 2-transitive.

So assume that $G$ is continuously 2 -transitive, then by Proposition 4.6 it is continuously 3 -transitive. If moreover $G$ is not continuously 4-transitive, then by Proposition 6.3 it is a convergence group and hence conjugate to a subgroup of $\operatorname{PSL}(2, \mathbb{R})$. On the other hand, since $G$ is continuously 3 -transitive, it is 3-transitive, and hence must be conjugate to the whole of $\operatorname{PSL}(2, \mathbb{R})$.

If we now assume that $G$ is continuously 4 -transitive then by Theorem 6.4 it is continuously $n$-transitive and hence $n$-transitive for every $n \in \mathbb{N}$. So if we take $f \in \operatorname{Homeo}\left(\mathbb{S}^{1}\right)$ and a finite set of points $x_{1}, \ldots, x_{n} \in \mathbb{S}^{1}$ there exists $g \in G$ such that $g\left(x_{i}\right)=f\left(x_{i}\right)$ and we are done. 
Theorem 7.2 Let $G$ be a closed transitive subgroup of Homeo $\left(\mathbb{S}^{1}\right)$ which contains a non constant continuous path. Then one of the following mutually exclusive possibilities holds:

(1) $G$ is conjugate to $\operatorname{SO}(2, \mathbb{R})$ in $\operatorname{Homeo}\left(\mathbb{S}^{1}\right)$.

(2) $G$ is conjugate to $\operatorname{PSL}_{k}(2, \mathbb{R})$ in $\operatorname{Homeo}\left(\mathbb{S}^{1}\right)$ for some $k \geq 1$.

(3) $G$ is conjugate to $\operatorname{Homeo}_{k}\left(\mathbb{S}^{1}\right)$ in $\operatorname{Homeo}\left(\mathbb{S}^{1}\right)$ for some $k \geq 1$.

Proof Since $G$ is a transitive subgroup of Homeo $\left(\mathbb{S}^{1}\right)$ which contains a non constant continuous path, Theorem 7.1 applies. It remains to show that if $G$ satisfies condition 3 in Theorem 7.1 then its closure is Homeo( $\left.\mathbb{S}^{1}\right)$.

To see this, let $f$ be an arbitrary element of $\operatorname{Homeo}\left(\mathbb{S}^{1}\right)$. If we can find a sequence of elements of $G$ which converges uniformly to $f$ then we shall be done. So let $\left\{a_{n}: n \in \mathbb{N}\right\}$ be a countable and dense set of points in $\mathbb{S}^{1}$. Choose a sequence of maps $g_{n} \in G$ so that $g_{n}\left(a_{k}\right)=f\left(a_{k}\right)$ for $1 \leq k \leq n$. Then $g_{n}$ will converge uniformly to $f$ so that the closure of $G$ will equal $\operatorname{Homeo}\left(\mathbb{S}^{1}\right)$.

Theorem 7.3 PSL $(2, \mathbb{R})$ is a maximal closed subgroup of Homeo( $\left.\mathbb{S}^{1}\right)$.

Proof Let $G$ be a closed subgroup of Homeo $\left(\mathbb{S}^{1}\right)$ containing $\operatorname{PSL}(2, \mathbb{R})$. Then $G$ is 3 -transitive and by applying Theorem 7.2 we can see that $\operatorname{Homeo}\left(\mathbb{S}^{1}\right)$ and $\operatorname{PSL}(2, \mathbb{R})$ are the only possibilities for $G$.

\section{References}

[1] M Bestvina, Questions in geometric group theory, collected by M Bestvina at http://www . math. utah.edu/ bestvina

[2] A Casson, D Jungreis, Convergence groups and Seifert fibered 3-manifolds, Invent. Math. 118 (1994) 441-456 MR1296353

[3] D Gabai, Convergence groups are Fuchsian groups, Ann. of Math. (2) 136 (1992) 447-510 MR1189862

[4] F W Gehring, G J Martin, Discrete quasiconformal groups. I, Proc. London Math. Soc. (3) 55 (1987) 331-358 MR896224

[5] É Ghys, Groups acting on the circle, Enseign. Math. (2) 47 (2001) 329-407 MR1876932

[6] A Hinkkanen, Abelian and nondiscrete convergence groups on the circle, Trans. Amer. Math. Soc. 318 (1990) 87-121 MR1000145 
[7] P Tukia, Homeomorphic conjugates of Fuchsian groups, J. Reine Angew. Math. 391 (1988) 1-54 MR961162

Mathematics Institute, University of Warwick

Coventry, CV4 7AL, UK

giblin@maths.warwick.ac.uk, markovic@maths.warwick.ac.uk

http://www . maths. warwick.ac.uk/ giblin/,

http://www.maths. warwick.ac.uk/ markovic/

Proposed: David Gabai

Seconded: Leonid Polterovich, Benson Farb
Received: 12 December 2005

Revised: 22 June 2006 